A N N A L E S Annales de Bretagne et des Pays de l'Ouest

\title{
Le massif granitique du Huelgoat. Pierres, carrières, constructions
}

Jean-Claude Meuret

\section{(2) OpenEdition}

Édition électronique

URL : https://journals.openedition.org/abpo/6905

DOI : $10.4000 / a b p o .6905$

ISSN : 2108-6443

Éditeur

Presses universitaires de Rennes

\section{Édition imprimée}

Date de publication : 30 juin 2021

Pagination : 210-213

ISBN : 978-2-7535-8359-7

ISSN : 0399-0826

\section{Référence électronique}

Jean-Claude Meuret, «Le massif granitique du Huelgoat. Pierres, carrières, constructions », Annales de Bretagne et des Pays de l'Ouest [En ligne], 128-2 | 2021, mis en ligne le 30 juin 2020, consulté le 10 octobre 2022. URL : http://journals.openedition.org/abpo/6905; DOI : https://doi.org/10.4000/abpo. 6905 
décennies du Xxe siècle, des travaux de restauration (on pourrait parler plus exactement de " rénovation ") mal conduits, en utilisant des matériaux industriels tout à fait inadaptés, ont aussi entraîné de nombreux dégâts, parfois irréparables, sur le bâti existant. La perte des savoir-faire anciens, le désamour du temps pour ce type de construction, le faible coût et la facilité d'obtention et d'emploi des matériaux industriels contemporains ont joué un rôle déterminant dans la destruction d'une part très importante de ce patrimoine. Celle-ci se poursuit malheureusement encore malgré les actions d'étude et de sensibilisation conduite par des institutions publiques ('Inventaire par exemple), parapubliques (les CAUE) ou des associations (Tiez Breiz en Bretagne ou Maisons paysannes de France ailleurs...). Le début du Xxi ${ }^{\mathrm{e}}$ siècle offre cependant quelques lueurs d'espoir. Il semble que la pédagogie patrimoniale conduite depuis la fin du siècle dernier ait commencé enfin à porter ses fruits. Elle a rejoint les nouvelles préoccupations environnementales et des évolutions dans les choix de cadres de vie de nos contemporains. Cela conduit à un net regain d'intérêt pour ce type de bâti ancien et à une reconsidération positive des techniques associées, qui se traduisent par des restaurations soucieuses de conserver l'authenticité des bâtiments et de renouer avec des traditions constructives anciennes respectueuses de l'intégrité des bâtiments. L'ouvrage nous en donne d'ailleurs deux beaux exemples (Launay Violet à Melesse et la Thébaudière à Montgermont).

Toutefois, ne nous illusionnons pas, ces belles restaurations restent minoritaires et nécessitent, dans les conditions économiques actuelles du secteur du bâtiment, soit de gros moyens financiers, soit l'investissement (lourd et de longue durée...) de la passion. En parallèle, l'effort de documentation du bâti en pan de bois ancien encore en élévation doit donc être plus que jamais maintenu, en usant de toutes les techniques disponibles aujourd'hui. Il s'agit souvent d'une lutte contre le temps, la célérité s'impose donc. Dans ce travail, le service de l'Inventaire joue un rôle majeur qu'il convient vraiment de souligner et de louer, aussi bien dans la recherche proprement dite que dans la vulgarisation des résultats de celle-ci, qui est indispensable pour une politique de préservation sur le long terme du bâti ancien.

Souhaitons pour conclure que ce petit livre très pédagogique, qui vise le plus grand nombre tout en offrant un propos de très grande qualité scientifique, sache attirer l'attention des décideurs (et financeurs...) publics, ainsi que des propriétaires, sur la nécessaire préservation de ce patrimoine en danger et qu'il puisse susciter de nouvelles vocations, tant pour la poursuite du travail de recherche engagé que pour la mise en chantier de nouvelles restaurations. La destruction, scandaleuse à plus d'un titre, au printemps 2020 d'une maison à pan de bois du XVII siècle dans le centre du village de Geudertheim (Bas-Rhin) par la mairie, alors que le bâtiment ne présentait aucune faiblesse structurelle, montre que le combat est encore loin d'être gagné...

Pierre-Yves LAFFONT

ChAURIS, Louis, Le massif granitique du Huelgoat. Pierres, carrières, constructions, Paris, Presses des Mines, collection sciences de la terre et de l'environnement, 2019, 152 p.

Excellent connaisseur du Massif armoricain, le géologue Louis Chauris, directeur de recherche au CNRS à la retraite vient de consacrer un ouvrage de 152 pages au massif granitique du Huelgoat, dans une série consacrée aux sciences de la terre et de l'environnement, publié aux Presses des Mines. Un tel travail pourrait paraître 
ne pas concerner directement le monde des historiens. Ce serait ignorer une part très imposante des publications de l'auteur dans lesquelles il expose sa science de la géologie, avant tout à des fins d'historique et d'archéologie du bâti, afin d'éclairer l'emploi des matériaux lithologiques dans l'Ouest, à toutes les époques où l'on en a usé. D'ailleurs, ses travaux ont très souvent été publiés dans des revues archéologiques et historiques de l'Ouest. Dans sa riche bibliographie, citons par exemple son ouvrage intitulé "Le kersanton, une pierre bretonne ", paru aux Presses Universitaires de Rennes en 2010.

Le présent ouvrage se subdivise en seize chapitres. Le premier traite logiquement des caractéristiques géologiques de cette entité du Huelgoat simplement limitée dans beaucoup de mémoires ou de publicités touristiques, aux images des mystérieux chaos granitiques. Il nous fait découvrir que le fameux granit (sans e pour les géologues) du Huelgoat s'inscrit dans une grande ligne de plutons -soit de grandes masses cristallines ovoïdes - armoricains qui barre d'ouest en est le nord de la péninsule, depuis Saint-Renan jusqu'à Dinan. Deux minéraux particuliers le caractérisent, la cordiérite et la tourmaline. Les cristaux du premier abondent, spécialement dans le granit au faciès porphyrique blanc-gris clair dit du Huelgat stricto sensu, le second a connu plusieurs associations et évolutions, générant ainsi nombre de variantes, tel le granit parfois plus foncé de Brennilis.

Au second chapitre, Louis Chauris traite des fameux " chaos " et " boules ", formes sous lesquelles ce granit apparait en surface et dans le paysage, et qui ont fait la célébrité esthétique et touristique de la région. Au-delà de nombreuses légendes, souvent celtiques, qu'ont inspirées ces formes surprenantes, il explique comment l'érosion d'énormes couches granitiques diaclasées, en dégradant en premier lieu les fissures, a généré les énormes boules. Leur usage par l'homme a commencé dans la mesure, avec les modestes prélèvements destinés à la création des mégalithes. La construction d'églises, à partir du second Moyen Âge, suscita des ponctions plus importantes, mais pour des usages encore très locaux. C'est au XIX siècle et jusqu'au $\mathrm{XX}^{\mathrm{e}}$ siècle que s'accrut fortement la pratique du débitage des boules les plus accessibles. On n'en était pas encore aux extractions profondes en carrières mais déjà, l'amputation du paysage traditionnel commença à susciter un mouvement de protection. Quelques grandes exploitations se développèrent, par exemple pour soutenir la reconstruction de Brest après la Seconde Guerre mondiale, mais presque toutes ont disparu à la fin du $\mathrm{xx}^{\mathrm{e}}$ siècle, en raison du recours généralisé et massif à d'autres matériaux que la pierre. Grâce à certains de ses nombreux clichés, l'auteur souligne la rapidité avec laquelle la végétation naturelle a repris ses droits sur les sites d'extraction. En 2018, seule subsiste la grande carrière profonde de RozPerez, permise par des moyens mécaniques surpuissants, et ce afin d'extraire une roche de haute qualité, à usage décoratif et funéraire, qui se vend jusqu'à l'étranger.

Au chapitre trois, à partir de recherches en archives, Louis Chauris, expose les problèmes sociaux et économiques suscités par les ouvertures de carrières dès 1825, lors du chantier du canal de Nantes à Brest. Carrières d'abord publiques, implantées selon certains responsables entrepreneurs et ingénieurs des Ponts et Chaussées "sur des landes incultes couvertes de rochers improductifs". Il nous semble qu'il révèle et entrouvre là un vaste thème de recherche encore un peu tabou, celui de la grave mutation rurale post-révolutionnaire qui vit en Bretagne, plus qu'ailleurs en France, les " communs des landes et terres gastes" basculer dans la propriété privée, alors qu'elles couvraient en ce début de XIX ${ }^{\mathrm{e}}$ siècle un tiers de l'espace breton et faisaient vivre des milliers de familles.

À ce point, l'auteur entame une suite de onze chapitres dans lesquels il passe en revue l'usage architectural du granit (sans e pour parler du matériau) depuis les monuments mégalithiques jusqu'à nos jours, soit sur près de sept millénaires. Ce 
n'est toutefois qu'à partir des chapelles et églises du XIVe siècle que dans les bourgs, le matériau commence vraiment à entrer dans le paysage architectural. Ayant régulièrement et assidûment fréquenté ces édifices, sans oublier croix, calvaires et autels, l'auteur en produit des descriptions souvent fines et précises, comme au Huelgoat, au Relecq, à Brennilis, Saint-Herbot, Pleyben, etc. On retient aussi ses conseils avisés pour ce qui est des matériaux utilisés lors des restaurations, pour lesquels on constate de plus en plus, et à regret, le recours à des matériaux totalement étrangers aux bâtiments et aux sites concernés.

Pour ce qui est des édifices laïques, les témoignages sont fréquemment tardifs, ce qui est bien exposé à partir des observations, mais aussi des archives pour les cas de Morlaix, Brest et son école navale, Carhaix ou Lorient au $\mathrm{Xx}^{\mathrm{e}}$ siècle, époque où le transport des matériaux lourds devient plus aisé. Ne peut-on cependant penser que l'examen des manoirs et châteaux, permettrait peut-être d'apporter des exemples plus anciens?

En ce même $\mathrm{xx}^{\mathrm{e}}$ siècle, la qualité et la réelle beauté du matériau lui permet de conquérir un nouveau créneau du bâti bien au-delà de son bassin géologique. Il s'agit d'abord des monuments aux morts après la Grande Guerre ainsi que des stèles commémoratives de faits militaires. Le granit du Huelgoat stricto sensu, et celui de Brennilis connurent ainsi une diffusion très large et surtout constamment visible au coeur de nombreuses agglomérations.

Il en est de même dans les cimetières de ce siècle, mais là plus spécialement pour le faciès de Brennilis, lequel permet un poli remarquable. Ce succès funéraire lui a même valu d'entrer dans l'imaginaire celtique sous la dénomination métaphorique de matériau de "la nuit celtique ". L'itinérance géologique de l'auteur lui a permis d'observer cet usage bien au-delà de la Bretagne, en Normandie, dans la région parisienne, dans le Nord. Cependant, l'expansion connaît maintenant un net recul en même temps que se répand la pratique de l'incinération.

Voirie, canal, viaducs et ponts ont eux aussi eu largement recours au granite porphyroïde du Huelgoat, ce dès le XIX ${ }^{\mathrm{e}}$ siècle. Le canal de Nantes à Brest fut l'initiateur, mais le chemin de fer prit le relais pour les gares, quais et viaducs tel celui de Châteauneuf-du-Faou ou ceux de Morlaix et Kerhuon lorsqu'il fallut panser les dégâts des bombardements de la Seconde guerre.

Dans les temps anciens, l'Armor, pays de la mer, n'a pas eu recours pour les aménagements portuaires et côtiers au granit porphyroïde du Huelgoat, car il en était encore trop éloigné. Là aussi, c'est surtout au $\mathrm{XX}^{\mathrm{e}}$ siècle que l'usage s'établit. Très tôt, le port de Brest le mit en œuvre pour les quais, parapets ou escaliers, puis il en fut de même à Lanvéoc-Poulnic, Camaret, l'Île de Batz... Plus tard, il n'est pas jusqu'au Sillon de Camaret, au port en eau profonde de Roscoff ou à l'embarcadère de Molène, en quête de lourds blocs pour faire face à l'océan, qui n'y aient eu recours.

L'enquête géologique et architecturale s'achève en la fameuse Vallée des Saints, en Carnoët (22) lieu du XXI ${ }^{\mathrm{e}}$ siècle qui se veut hautement inscrit dans le passé spirituel et mythique de la Bretagne. Sans entrer dans les réprobations ou enthousiasmes que suscite cette " Ille de Pâques bretonne ", Louis Chauris souligne le large usage par les sculpteurs de la pierre du Huelgoat, précisant que la plus haute statue, celle de saint Nikodem, est née d'un bloc du faciès de Brennilis, et qu'avec $5,80 \mathrm{~m}$, elle se place parmi les plus hautes.

Avec modestie, l'auteur reconnaît que son enquête sur l'usage des granites du Huelgoat, demeure bien incomplète pour de nombreuses régions de France, mais aussi pour l'étranger. Il souhaite que les éclairages et les pistes qu'il propose au lecteur soient autant de "pénétrantes " pour les chercheurs à venir. Ajoutons qu'en ces temps où triomphent l'instantané et le virtuel et où s'impose le " distanciel ", on ne peut que saluer le bel exemple de son travail, fondé sur des recherches conti- 
nues, réelles et incessantes, in situ dans les carrières, les ports, les édifices, mais aussi aux archives, et de plus rédigé en synthèses accessibles, même aux non géologues que nous sommes le plus souvent.

Jean-Claude MEURET

Hoareau, Muriel, Poton de Xaintrailles, Didier, et Pairault, Louis-Gilles (dir.), Libraires et imprimeurs protestants de la France atlantique, XVI ${ }^{\ell}-X V I I^{e}$ siècle, Rennes, Presses Universitaires de Rennes, 2020, 176 p.

L'histoire de l'imprimerie française sous l'Ancien Régime a privilégié les grands centres d'édition qu'étaient Paris, Lyon et Rouen. Elle néglige parfois les régions situées dans l'Ouest du royaume, de la Basse-Normandie au Béarn, où les communautés protestantes ont développé des centres d'imprimerie dans des villes petites et moyennes comme La Rochelle, Saumur, Niort, Montauban, Sainte-Foy-la-Grande, Orthez ou Puylaurens.

Les neuf articles réunis par Muriel Hoareau, Didier Poton de Xaintrailles et Louis-Gilles Pairault sont des contributions qui ont été présentées pendant les journées d'études organisées les 9 et 10 novembre 2017 à La Rochelle par le Centre de Recherche en Histoire Internationale et Atlantique, à l'occasion de la commémoration du cinq-centième anniversaire de la Réforme protestante. La Rochelle ayant été un des pôles majeurs de la librairie et de l'imprimerie protestantes jusqu'en 1628, elle était le lieu idéal pour réunir des spécialistes de l'histoire du livre et du protestantisme dans la France atlantique (Didier Boisson, Jean-Claude Bonnin, Philippe Chareyre, Thomas Guillemin, Muriel Hoareau, Marielle Mouranche, Jean-Paul Pittion, Didier Poton de Xaintrailles, Éric Suire).

Cette étude souligne les caractéristiques de l'édition protestante dans l'Ouest de la France, où les imprimeries eurent une activité plus irrégulière que dans le reste du pays, voire intermittente. Elle souligne d'abord l'évolution du centre de gravité de la production imprimée protestante. Le cœur de l'imprimerie huguenote fut d'abord La Rochelle, pendant une trentaine d'années à partir de 1568, date à laquelle le port saintongeais devint la capitale du parti huguenot. Les presses rochelaises de Barthélemy Berton et de Jean Portau, et celles de la dynastie des Haultin, défendirent la Cause avec conviction. Ensuite, de 1600 environ à 1685, l'imprimerie saumuroise s'imposa au détriment de celle de La Rochelle.

Les auteurs des communications soulignent ensuite la vie itinérante des imprimeurs. Thomas Portau imprima pour la cause huguenote à Pons et à Niort avant de s'installer à Saumur en 1601 ; Pierre Haultin arriva à La Rochelle à la demande de Jeanne de Navarre après avoir vécu à Paris et à Lyon ; Jérôme Haultin séjourna plus de dix ans en Angleterre avant de s'installer à La Rochelle, et Denis Haultin partit à Montauban en 1589. Dans le Sud-Ouest, les ateliers d'imprimerie étaient mobiles et les entrepreneurs se déplaçaient constamment avec une presse et un à deux jeux de caractères.

Les logiques éditoriales furent en partie imposées par le contexte culturel. L'absence d'université et d'académie protestante à La Rochelle conduisit les imprimeurs à produire des livres destinés surtout à l'ensemble de la France et à l'exportation. En revanche, la présence de l'académie et du collège protestants à Saumur explique la réputation des imprimeurs huguenots de cette ville, dont les plus renommés travaillaient pour l'académie : Thomas Portau, Jean Lesnier, Isaac Desbordes, Henri Desbordes. De même, c'est la présence d'institutions scolaires et 